\title{
Mitochondrial Dysfunction in Traumatic Brain Injury: Management Strategies
}

\author{
Carlos-Andrés Bonilla-Mendoza ${ }^{1}$ Ezequiel Garcia-Ballestas ${ }^{1}$ Alfonso Pacheco-Hernandez ${ }^{2}$ \\ Luis-Rafael Moscote-Salazar ${ }^{3}$ Ravish R. Keni ${ }^{4}$ Amit Agrawal ${ }^{5}$
}

${ }^{1}$ Centro de Investigaciones Biomédicas (CIB), Faculty of Medicine, University of Cartagena, Cartagena, Columbia

${ }^{2}$ Fundación Centro Colombiano de Epilepsia y Enfermedades

Neurologicas-FIRE, Faculty of Medicine, University of Cartagena, Cartagena, Columbia

${ }^{3}$ Centro de Investigaciones Biomedicas (CIB), Cartagena

Neurotrauma Research Group Research Line, Faculty of Medicine, University of Cartagena, Cartagena, Columbia

${ }^{4}$ Department of Neurology, Narayana Medical College and Hospital,

Nellore, Andhra Pradesh, India

${ }^{5}$ Department of Neurosurgery, Narayana Medical College and

Hospital, Nellore, Andhra Pradesh, India

Indian J Neurotrauma:2020;17:37-41

\begin{abstract}
Address for correspondence Amit Agrawal, MCh, Department of Neurosurgery, Narayana Medical College Hospital, Chinthareddypalem, Nellore 524003, Andhra Pradesh, India (e-mails: dramitagrawal@gmail.com; dramitagrawal@hotmail.com).
\end{abstract}

\begin{abstract}
Keywords

- mitochondria

- traumatic brain injury

- apoptosis

Today, traumatic brain injuries continue to be studied, increasingly investigating the pathophysiological mechanisms that contribute to the clinical presentation, severity, and possible sequelae, but despite this, the prognosis of these patients is sometimes poor. Mitochondrial dysfunction comprises a series of reactions that contribute to the inflammatory process in these patients that have an impact on the prognosis, since it is one of the pathophysiological mechanisms involved in secondary lesions after a traumatic brain injury; and therefore has opened a field of study in the search of possible biomolecular markers that allow us to establish a prognosis and prediction of mortality.
\end{abstract}

\section{Introduction}

Nowadays, the biggest concerns in traumatic brain injury (TBI) are secondary injuries, where edema and the release of chemical substances promote the inflammatory process, cellular injury, or cell death. The high morbidity and mortality are mainly due to the lack of effective diagnoses and treatments despite the intensive care strategies for these patients. ${ }^{1}$ An important limitation for a successful outcome is the lack of understanding of the underlying complex and dynamic cellular and molecular events, the characterization of a cascade of biochemical and pathophysiological reactions, ${ }^{2,3}$ emphasizing mitochondrial dysfunction, key to investigate more deeply about this pathology in the search of possible biomarkers that allow an opportune diagnosis, stratification of the severity, monitoring, and prognosis, with this identifying possible complications, and the opportunity to establish better strategies before these cases. ${ }^{3}$ This article will review the cellular processes that are performed after a TBI, specifically in the mechanisms of mitochondrial dysfunction, the way in which they affect the course of the disease, and the measures currently used in this situation.

\section{Traumatic Brain Injury}

TBI is one of the main causes of morbidity and mortality worldwide. ${ }^{4,5}$ It is a pathology that generates more than 2.5 million visits to emergencies and controls every year ${ }^{4,6}$ and has an overall mortality rate of 19 per 100,000 inhabits. ${ }^{6}$ It is characterized by neurological alterations resulting from a traumatic head injury whose clinical assessment may include conscience disorders, neurophysiological impairment, bony fractures, and/or intracranial injuries attributable to the traumatic impact. ${ }^{6}$ These brain alterations occur due to the following two important pathophysiological processes ( - Fig. 1): (1) primary injury being the result of the 


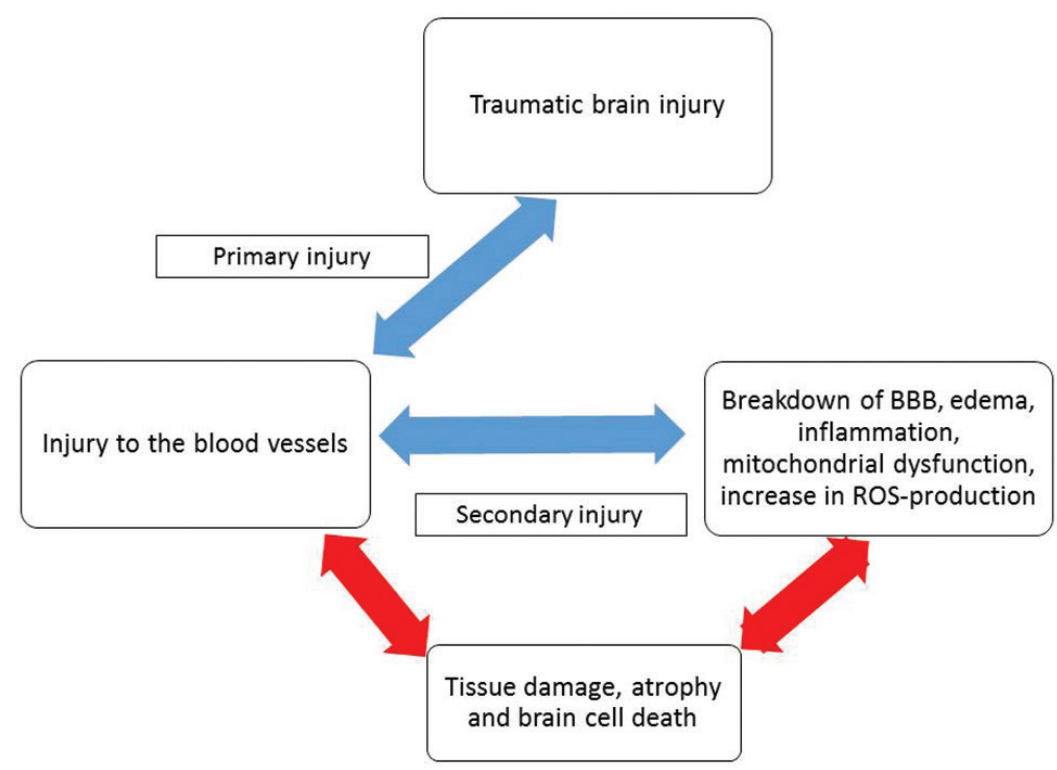

Fig. 1 Pathophysiology of brain alterations after neurotrauma.

mechanical impact depending on the relationship with the mechanism and the transferred energy reaching cell damage, tearing and axonal retractions, and vascular alterations; ;,6 and (2) the secondary injury which happens due to the effects of the primary injuries prompting several molecular, inflammatory, and metabolic processes that lead to the release of excitotoxic neurotransmitters, alteration in the permeability of the membrane, promoting the entry of calcium and potassium exit, and mitochondrial dysfunction, triggering cell death by a process of necrosis and/or apoptosis. . $6^{4}$

\section{Pathology}

Free radicals, which under physiological conditions are involved in inflammatory processes, as well as in learning and memory, ${ }^{5}$ its excessive production induces a state of oxidative stress carrying out the progressive deterioration of proteins, alteration in the permeability of the cellular membrane as mitochondrial, alteration of signaling pathways, and damage to the genetic material. ${ }^{7-9}$ In TBI, clinically, biomarkers characteristic of mitochondrial dysfunction can be identified after 30 minutes, which indicates an increase in proton conductance through the inner membrane of the mitochondria, in turn indicating the onset of a mitochondrial permeability transition; ${ }^{10}$ and thus, the increase in mitochondrial $\mathrm{Ca}^{2+}$ uptake overwhelms the mitochondria causing the activation of nitric oxide synthase (NOS) inducing a mitochondrial production of nitric oxide (NO), as well as the escape of superoxide radicals $\left(\mathrm{O}_{2}\right)$. These two free radicals rapidly react with each other to form a peroxynitrite (PN) anion that reacts with carbon dioxide to generate more free radicals, responsible for activating a series of pathological processes which will trigger oxidative damage mediated by the peroxidation of susceptible lipids (polyunsaturated fatty acids) of the mitochondrial membrane and inhibition of the electron chain. ${ }^{1,2,411} \mathrm{PN}$ is the main substance responsible for tissue insult. As this oxidative damage progresses within the mitochondria, it causes the loss of mitochondrial membrane potential, the respiratory chain is seriously compromised, drastically limiting the production of ATP, and ultimately, the transition of mitochondrial permeability ( $\mathrm{mPt}$ ) that worsens mitochondrial damage and the subsequent cytosolic involvement. ${ }^{5,9,11}$

When this occurs, the $\mathrm{Ca}^{2+}$ which was absorbed by the mitochondria is released back into the cytoplasm (due to the involvement of the mPT), exacerbating the activation of proteases activated by $\mathrm{Ca}^{2+}$, such as calpain and caspase 3 (-Fig. 2), which causes protein proteolysis of the neuronal cytoskeleton. ${ }^{9,11}$ In addition, PN anions are highly reactive and can react with more carbon dioxide to form nitrosoperoxycarbonate (ONOOCO2) which may injure other components, such as endoplasmic reticulum and the cell membrane, whose polyunsaturated fatty acids are susceptible to peroxidation in comparison with other tissues of the body, making the functional integrity of the neuronal cell more vulnerable. ${ }^{11}$ In humans, the development of these complex events in TBI by the decrease in the respiratory rate and less production of ADT with an augmented use of oxygen has been demonstrated. ${ }^{12}$

\section{Role of Inflammatory Response}

The purpose of all inflammatory processes is to induce apoptosis, which is mediated by the inflammatory process that is performed in the tissue by the action of all mentioned free radicals, producing mitochondrial deterioration which increases the permeability of the organelle membrane and favoring the release of a variety of enzymes and compounds that induce apoptotic signaling pathways; these apoptotic effectors released by mitochondria activate caspases, proteins whose objective is to break down the cellular structures necessary for cell survival. In the same way, cytochrome $\mathrm{C}$ 


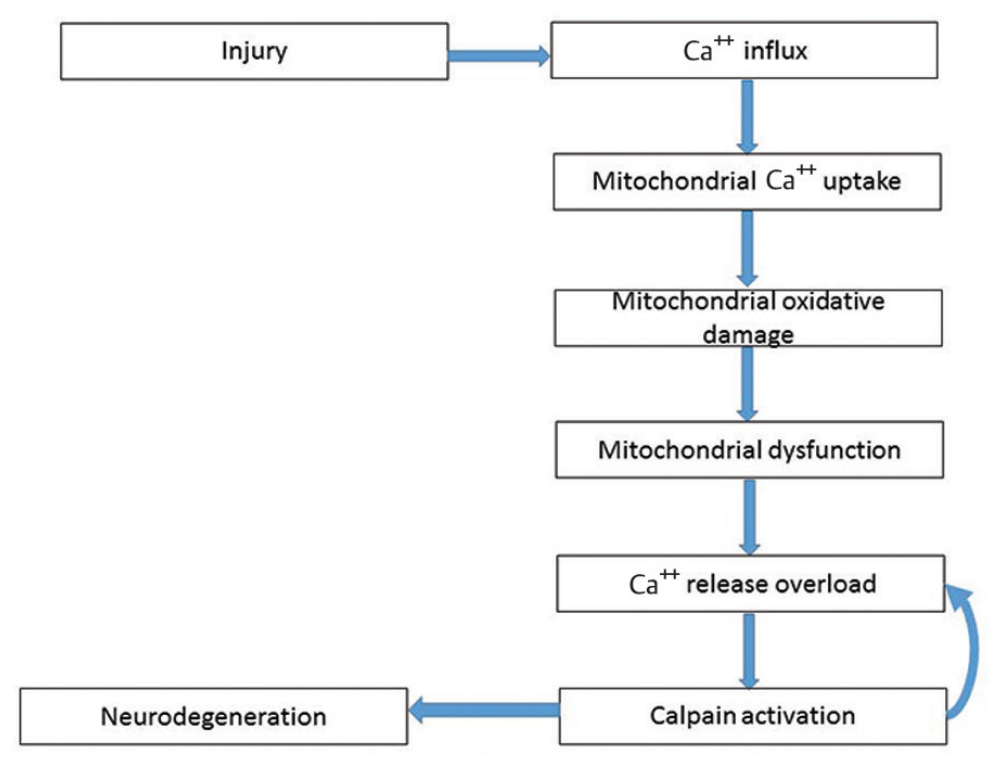

Fig. 2 Mitochondrial oxidative dysfunction and neurodegeneration after neurotrauma.

is released, which will bind with the activation factor of the apoptotic protease (Apaf-1) and ATP to form a protein complex called apoptosome..$^{2-4}$ These apoptosomes adhere to caspase-activating proteins. The direct initiation of apoptotic mechanisms arises from the activation of the first signaling process of apoptosis ligand and tumor necrosis factor (TNF), which is the main extrinsic mediator of apoptosis activated by macrophages. It is important to detail that altogether neurotransmitters of excitatory amino acids (glutamate) are subsequently released that affects the neurons and also fulfills different physiopathological processes. Continuous excitotoxic cell damage and inflammation will lead to necrosis and apoptosis., ${ }^{411}$

Another important process in the mitochondrion is the imbalance of fission and fusion which have been associated with mitochondrial dysfunction. Mitochondrial fusion induces the fragmentation of mitochondria from a tubular morphology into pieces; in contrast, perturbed mitochondrial fission results in the fusion of adjacent mitochondria. ${ }^{13}$ In other words, an imbalance of mitochondrial fission and fusion, particularly an excess of fission, can be detrimental to mitochondrial function, causing decreased respiration, reactive oxygen species (ROS) production, and apoptosis. The translocation of Dynamin-related protein 1 (Drp1) to the mitochondrial outer membrane (OM) is critical to the development of this process. ${ }^{14}$

\section{Role of Mitochondria in TBI}

Mitochondria are organelles distributed in the cytosol of neurons and have the function of supplying most energy substrates in the form of ATP (main source of energy for most cellular functions), ${ }^{2,4}$ approximately 95\% through oxidative phosphorylation processes which is vital for proper neuronal functioning. ${ }^{2}$ Its structural configuration is given by a dual membrane (internal and external) separated by the intermembrane space, each responsible for specific functions. The OM contains transporter proteins, such as the voltagedependent anion channel, as well as the translocases, which have the imperative function of importing and exporting a variety of ions and proteins to maintain mitochondrial function. ${ }^{4,11}$ Within this, membrane can find internal proteins of the mitochondrial membrane essential in the cellular respiration process, such as complex I, complex III, complex IV, ATP synthase, the transporter of adenosine nucleotides (ANT), and calcium and sodium exchangers., ${ }^{2,411}$ The phospholipids of their membranes play an important role in cell structure and survival using a role in the moderation of cell proliferation as programmed cell death. ${ }^{3-5}$

At the cellular level, mitochondria modulate transmembrane potential, cellular thermal regulation and calcium storage, ${ }^{1,4}$ an essential component in bioenergy, and cellular function; they also have the task of maintaining the homeostasis of neuronal cells through a variety of intricate processes, such as autophagy, the elimination of compounds and harmful elements of metabolism, in response to a variety of metabolic demands that include the stress associated to ROS. ${ }^{4,9}$

\section{Management Strategies}

A promising treatment would be the reduction of the free radicals that are in excess, thus allowing a decrease in oxidative stress that causes damage and neuronal degeneration. The antioxidant therapy is a strategy whose purpose is the use of complementary antioxidants that could sweep away free radicals. ${ }^{4,15}$ Given the great evidence of the high morbidity and mortality rates of TBI, as aforementioned, it is more valuable to have measures or therapeutic strategies that allow us to have preventive procedures which could reduce the symptoms and neurological injuries as a result of the trauma. Research studies provide evidence that mitochondrial dysfunction plays a dominant role in the pathophysiology of TBI, 
so the product of current research reflects some agents that can modulate mitochondrial damage. The $\mathrm{O}_{2}$ supplementation therapy turned out to be a neuroprotective therapy because the oxygen preserves the structure of the mitochondrial membrane, but nevertheless, prolonged use exposes to a risk of increased production of oxygen radicals, exacerbating neuronal dysfunction and subsequent apoptosis, ${ }^{4}$ so the indications are that current available and more rigorous clinical studies are recommended.

\section{Target Molecules}

SS-31 (D-Arg-Dmt-Lys-Phe-NH2; Dmt-2',6'-dimethyltyrosine) is a novel mitochondria-targeted peptide which can penetrate the inner membrane of the mitochondria and have a great antioxidant effect. Zhu et al demonstrated in rat-based models that the administration of SS-31 led to reduced edema, oxidative stress, total burden of ROS, iron overload which is cytotoxic due to its ability to create free radicals, and reduction in the indicators of DNA alterations by ROS; and moreover, they observed an increase of antiapoptotic biomarkers, and also, nuclear translocation of PGC- $1 \alpha$ that is a promoter of mitochondrial function and biogenesis. ${ }^{16}$ Recently, the evidence has showed up the administration of mitochondrial division inhibitor-1 (Mdivi-1), an allosteric inhibitor of the translocation of Drp1, through the reduction in the binding affinity with Guanosine triphosphate (GTP) in a critical step in the mitochondrial fusion has some encouraging and promising favorable effects in the TBI management. Fischer et al demonstrated that when Mdivi-1 was administered after a TBI in rat-based models, there was no expression of changes attributable to mitochondrial dysfunction, such as loss of neurons, increased size of mitochondrion, and cognitive deficit resulting from TBI. ${ }^{14}$

There are some management strategies which have shown a great influence in the outcome of individuals, among them Coenzyme Q10, the most powerful, demonstrates an effective reduction of oxidative stress. ${ }^{4,15}$ An important endogenous antioxidant is ubiquinol, a reduced form of coenzyme Q10 stabilizes the membranes, especially the inner mitochondrial membrane which prevents mitochondrial dysfunction by stabilizing them. It also improves energy production and biogenesis processes, as well as inhibit cell death through mitochondrial pathways. ${ }^{15} \mathrm{~N}$-acetyl cysteine is another molecule which possesses antiapoptotic properties, and improves mitochondrial energy by increasing glutathione., ${ }^{2,4}$ Another interesting strategy is cyclosporin A (CsA), which has been shown to inhibit the increase of mitochondrial permeability and decrease the formation of free radicals. ${ }^{2,17}$ It also intervenes in the inhibition of $\mathrm{Ca}^{2+}$ entry in mitochondria. ${ }^{18}$

These drugs have shown improvement in the results once administered 24 hours after the injury. Although it is necessary to note that the most important therapeutic interval in which treatment would result useful are the first 3 hours, therefore, any treatment established after these time will protect against the late insult but will not prevent the insult of early phase. ${ }^{10}$ These data suggest that the drugs that protect the mitochondria have long therapeutic windows, so it is still reliable to administer them hours after the event, obtaining a therapeutic efficacy. ${ }^{18}$ Contemplating the functions could be a complementary treatment to reduce the severity of secondary injury after trauma. ${ }^{15,18}$

\section{Conclusion}

Mitochondrial biomarkers are a very important tool in TBI like a prognostic factor and very indicative or mortality predictor; even if the patient survive, the secondary damage will be permanent. When there is structural damage in the neuron, the increase of $\mathrm{Ca}^{2+}$ will inevitably end up in the increase of free radicals within the neuron and the apoptosis. In some animal models, the evaluation of mitochondrial DNA has proved to be an important biomarker in the prognosis and follow-up of patients with TBI that shows a dramatic increase in the 25 hours, following trauma. There is a need to develop neuroprotective measures capable of reducing the amount of these effects if necessary. However, to date there is no Food and Drug Administration (FDA)-approved treatment for this disease, ${ }^{2}$ which means that doctors today are willing to only provide palliative treatments that may not be enough after all. There are some strategies like the peptide SS-31 to reduced edema and the oxidative stress, N-acetyl cysteine that has antiapoptotic properties, and the CsA that has shown to decrease the formation of free radicals. There are promising studies in animal models about mitochondrial pathophysiology in TBI and the tools that it provides us as biomarkers (mitochondrial DNA), and the possibility of blocking oxidative stress as a tool to improve the prognosis in TBI.

\section{Conflict of Interest}

None.

\section{References}

1 Wang WX, Sullivan PG, Springer JE. Mitochondria and microRNA crosstalk in traumatic brain injury. Prog Neuropsychopharmacol Biol Psychiatry 2017;73:104-108

2 Yonutas HM, Vekaria HJ, Sullivan PG. Mitochondrial specific therapeutic targets following brain injury. Brain Res 2016; 1640(Pt A) :77-93

3 González-Domínguez R. Medium-chain fatty acids as biomarkers of mitochondrial dysfunction in traumatic brain injury. EBioMedicine 2016;12:8-9

4 Hiebert JB, Shen Q, Thimmesch AR, Pierce JD. Traumatic brain injury and mitochondrial dysfunction. Am J Med Sci 2015; 350(2):132-138

5 Dobrachinski F, da Rosa Gerbatin R, Sartori G, et al. Regulation of mitochondrial function and glutamatergic system are the target of guanosine effect in traumatic brain injury. J Neurotrauma 2017;34(7):1318-1328

6 Charry JD, Cáceres JF, Salazar AC, et al. Trauma craneoencefálico. Revisión de la literatura. Rev Chil Neurocir. 2017;43:177-182

7 Grimm A, Eckert A. Brain aging and neurodegeneration: from a mitochondrial point of view. J Neurochem 2017;143(4): 418-431 
8 Saha P, Gupta R, Sen T, Sen N. Activation of cyclin D1 affects mitochondrial mass following traumatic brain injury. Neurobiol Dis 2018;118:108-116

9 Zorov DB, Juhaszova M, Sollott SJ. Mitochondrial reactive oxygen species (ROS) and ROS-induced ROS release. Physiol Rev 2014;94(3):909-950

10 Singh IN, Sullivan PG, Deng Y, Mbye LH, Hall ED. Time course of post-traumatic mitochondrial oxidative damage and dysfunction in a mouse model of focal traumatic brain injury: implications for neuroprotective therapy. J Cereb Blood Flow Metab 2006;26(11):1407-1418

11 Hill RL, Singh IN, Wang JA, Hall ED. Time courses of post-injury mitochondrial oxidative damage and respiratory dysfunction and neuronal cytoskeletal degradation in a rat model of focal traumatic brain injury. Neurochem Int 2017;111:45-56

12 Verweij BH, Muizelaar JP, Vinas FC, Peterson PL, Xiong Y, Lee CP. Impaired cerebral mitochondrial function after traumatic brain injury in humans. J Neurosurg 2000;93(5):815-820
13 Hu C, Huang Y, Li L. Drp1-dependent mitochondrial fission plays critical roles in physiological and pathological progresses in mammals. Int J Mol Sci 2017;18(1):144

14 Fischer TD, Hylin MJ, Zhao J, Moore AN, Waxham MN, Dash PK. Altered mitochondrial dynamics and TBI pathophysiology. Front Syst Neurosci 2016;10:29

15 Venegoni W, Shen Q, Thimmesch AR, Bell M, Hiebert JB, Pierce JD. The use of antioxidants in the treatment of traumatic brain injury. J Adv Nurs 2017;73(6):1331-1338

16 Zhu Y, Wang H, Fang J, et al. SS-31 provides neuroprotection by reversing mitochondrial dysfunction after traumatic brain injury. Oxid Med Cell Longev 2018;2018:4783602

17 Millet A, Bouzat P, Trouve-Buisson T, et al. Erythropoietin and its derivates modulate mitochondrial dysfunction after diffuse traumatic brain injury.J Neurotrauma 2016;33(17):1625-1633

18 Mohamadpour M, Whitney K, Bergold PJ. The importance of therapeutic time window in the treatment of traumatic brain injury. Front Neurosci 2019;13:07 